\title{
Rapid dramatic improvement of pustulotic arthro-osteitis by guselkumab in a patient with palmoplantar pustulosis: a real-world experience
}

\author{
Nobuyuki Kikuchi, Toshiyuki Yamamoto
}

Department of Dermatology, Fukushima Medical University, Fukushima 960-1295, Japan

Corresponding author: Prof. Toshiyuki Yamamoto, E-mail: toyamade@fmu.ac.jp

Sir,

A 59-year-old female with palmoplantar pustulosis (PPP) of 20 years' duration was referred to our hospital. Although she had been treated with topical corticosteroid ointment, skin lesions had waxed and waned during this time period. She was a past smoker (three cigarettes a day for 1 year), but quit smoking soon after she was diagnosed as PPP. She occasionally had a sore throat when she was a child. Her mother also had PPP. The patient further developed arthralgia involving the left clavicle two years previously. Physical examination showed palmoplantar erythema with scales and a small number of pustules and vesiculopustules (Fig. 1). Skin atrophy due to long-term topical corticosteroid therapy was also observed. She did not have acne. Laboratory examination showed slight increases in inflammatory markers (C-reactive protein; $2.0 \mathrm{mg} / \mathrm{dl}$, erythrocyte sedimentation rate; $34 \mathrm{~mm} / \mathrm{h}$ ); however, anti-thyroid, microsome, and nuclear antibodies were all within normal limits. Examination using technetium-99m bone scintigraphy revealed increased uptake in the left clavicle (Fig. 2). Otolaryngological examination revealed tonsillar hypertrophy. For joint pain, non-steroidal anti-inflammatory drugs (NSAIDs), methotrexate (6 mg/week) for 10 months, and cyclosporine (100-200 mg/day) for 8 months were administered, all of which resulted in little effect. The patient refused tonsillectomy, and guselkumab was initiated for severe joint pain. A few days after the first subcutaneous injection of guselkumab (100 mg), the patient's joint pain was dramatically improved, and NSAIDs were not needed. Her visual analogue scale score for joint pain was decreased to 0 .
Joint manifestation is a major comorbidity of PPP, and is known as pustulotic arthro-osteitis (PAO) [1]. The clavicles, sternum, and sternoclavicular joints are mostly affected, but peripheral joints are also sometimes involved. PAO is also triggered by focal infection. Previous studies have shown that PAO occurs in around 20 to $30 \%$ of all PPP patients [2]. To date, there are few satisfactory therapies for PAO, which severely impairs the patient's quality of life. Patients with PPP have difficulties in daily activities requiring the use of hands and feet, such as routine daily work, professional work, and walking, as well as cosmetic concerns. Furthermore, PAO patients have a high burden of illness.

NSAIDs, methotrexate, cyclosporine, potassium iodide, bisphosphonate, and antibiotics have been used in the treatment of PAO; however, conventional therapies have been disappointing in many cases. Recently, biologics may be a good candidate for refractory cases of PPP, although to date, reports on the efficacy are limited. There are several studies of ustekinumab, alefacept, or combination therapy with adalimumab and ustekinumab for refractory and/or severe PPP [3-5]. Recently, guselkumab, an antiIL-23 monoclonal antibody, was reported to significantly reduce the mean PPP severity index total scores from baseline at week 16 in 25 patients with PPP, and the improvement was maintained through 24 weeks [6]. Biologics may be an effective new therapy for PPP; however, their effects on PAO are largely unknown, and only a few case reports have been reported [7].

Our patient occasionally had a sore throat when she was young. Tonsillectomy was therefore recommended,

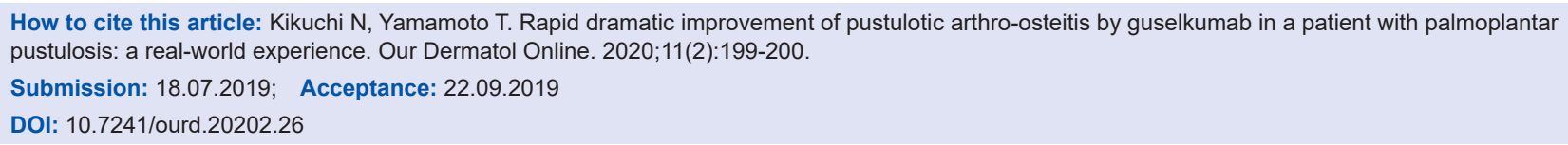




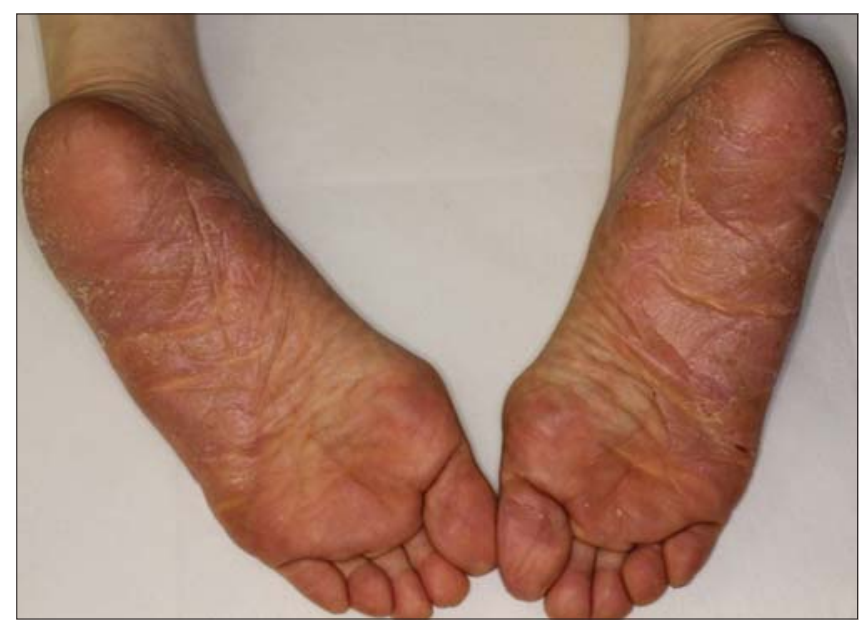

Figure 1: Diffuse erythema, scales, and a small number of tiny pustules on the bilateral soles.

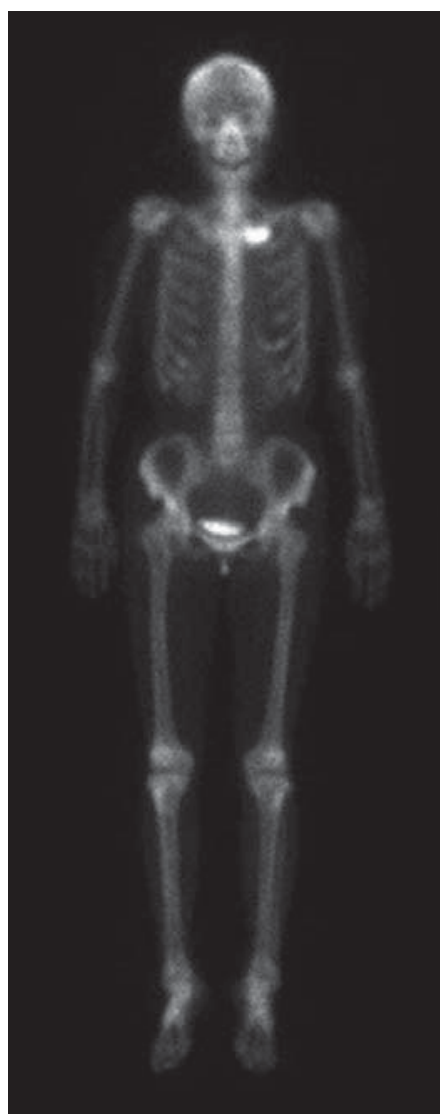

Figure 2: Bone scintigraphy showing increased uptake in the left clavicle. but refused by the patient, and the joint manifestations rapidly progressed. Therapies with methotrexate and cyclosporine were not sufficiently effective. We then administered guselkumab, which was recently approved for insurance coverage in Japan. Only a single administration of guselkumab resulted in a dramatic effect on the patient's severe joint pain. Clinical trials have demonstrated that effects of guselkumab on PPP skin lesions were gradual, not rapid [6]. Thus, the patient of the current report is still on a regimen of guselkumab therapy (100 mg subcutaneously administered at weeks 0 and 4 , and every 2 months thereafter). Furthermore, the sustained effects of guselkumab for joint manifestation have been carefully monitored.

\section{Consent}

The examination of the patient was conducted according to the Declaration of Helsinki principles.

\section{REFERENCES}

1. Yamamoto T. Pustulotic arthro-osteitis associated with palmoplantar pustulosis. J Dermatol. 2013;40:857-63.

2. Yamamoto T. Clinical characteristics of Japanese patients with palmoplantar pustulosis. Clin Drug Invest. 2019;39:241-52.

3. Gerdes S, Franke J, Domm S, Mrowietz U. Ustekinumab in the treatment of palmoplantar pustulosis. Br J Dermatol. 2010;163:1116-8.

4. Guenther LC. Alefacept is safe and efficacious in the treatment of palmoplantar pustulosis. J Cutan Med Surg. 2007;11:202-5.

5. Torre KM, Payette MJ. Combination biologic therapy for the treatment of severe palmoplantar pustulosis. JAAD Case Rep. 2017;3:240-2.

6. Terui T, Kobayashi S, Okubo Y, Murakami M, Hirose K, Kubo H. Efficacy and safety of guselkumab, an anti-interleukin 23 monoclonal antibody, for palmoplantar pustulosis: a randomized clinical trial. JAMA Dermatol. 2018;154:309-16.

7. Ohashi T, Suzuki Y, Yamamoto T. Use of biologics for pustulotic arthro-osteitis in two patients with palmoplantar pustulosis. J Dermatol. 2017;44:97-8.

Copyright by Nobuyuki Kikuchi, et al. This is an open-access article distributed under the terms of the Creative Commons Attribution License, which permits unrestricted use, distribution, and reproduction in any medium, provided the original author and source are credited.

Source of Support: Nil, Conflict of Interest: None declared. 